\title{
NUMERICAL INDEX OF BANACH SPACES AND DUALITY
}

\author{
Konstantin Boyko, Vladimir Kadets, \\ Faculty of Mechanics and Mathematics \\ Kharkov National University \\ pl. Svobody 4, 61077 Kharkov, Ukraine \\ E-mail: k_boyko@ukr.net, vovalkadets@yahoo.com \\ Miguel Martín ${ }^{1}$, \\ Departamento de Análisis Matemático \\ Facultad de Ciencias \\ Universidad de Granada \\ 18071 Granada, Spain \\ E-mail: mmartins@ugr.es \\ AND DIRK WERNER \\ Department of Mathematics \\ Freie Universtät Berlin \\ Arnimallee 2-6, D-14195 Berlin, Germany \\ E-mail: werner@math.fu-berlin.de
}

\begin{abstract}
We present an example of a Banach space whose numerical index is strictly greater than the numerical index of its dual, giving a negative answer to a question which has been latent since the beginning of the seventies. We also show a particular case in which the numerical index of the space and the one of its dual coincide.
\end{abstract}

Date: August 1st, 2005.

2000 Mathematics Subject Classification. Primary: 46B20, 47A12. Secondary: 46B22, 46E15.

Key words and phrases. Numerical range; numerical radius; numerical index; duality; C-rich subspaces.

The work of the second-named author was supported by a fellowship from the Alexander-von-Humboldt Stiftung. The work of the third-named author was partially supported by Spanish MCYT project no. BFM2003-01681 and Junta de Andalucía grant FQM-185.

${ }^{1}$ Corresponding author. 


\section{InTRODUCTION}

The concept of numerical index of a Banach space was first suggested by G. Lumer in 1968 (see [6]); it is a parameter relating the norm and the numerical range of operators on the space. The notion of numerical range was first introduced by O. Toeplitz in 1918 36 for matrices, and it was extended in the sixties to bounded linear operators on an arbitrary Banach space by F. Bauer 1] and G. Lumer 21. Classical references here are the monographs by F. Bonsall and J. Duncan [3, 4. For recent results we refer the reader to 8, 9, 10, 18, 23, 25, 26, 30, 33], and to the expository paper [22] and references therein.

Here and subsequently, for a real or complex Banach space $X$, we write $B_{X}$ for the closed unit ball and $S_{X}$ for the unit sphere of $X$. The dual space is denoted by $X^{*}$, and the Banach algebra of all bounded linear operators on $X$ by $L(X)$. The numerical range of such an operator $T$ is the subset $V(T)$ of the scalar field defined by

$$
V(T):=\left\{x^{*}(T x): x \in S_{X}, x^{*} \in S_{X^{*}}, x^{*}(x)=1\right\} .
$$

The numerical radius of $T$ is the seminorm defined on $L(X)$ by

$$
v(T):=\sup \{|\lambda|: \lambda \in V(T)\}
$$

for each $T \in L(X)$. The numerical index of the space $X$, is the constant $n(X)$ defined by

$$
n(X):=\inf \{v(T): T \in L(X),\|T\|=1\}
$$

or, equivalently, the greatest constant $k \geqslant 0$ such that $k\|T\| \leqslant v(T)$ for every $T \in L(X)$. Note that $0 \leqslant n(X) \leqslant 1$, and $n(X)>0$ if and only if $v$ and $\|\cdot\|$ are equivalent norms on $L(X)$ (the numerical radius can be a non-equivalent norm on $L(X)$; see [28, Example 3.b]). In the complex case, it is a celebrated result due to H. Bohnenblust and S. Karlin [2] (see also 12 ) that $n(X) \geqslant 1 / \mathrm{e}$, so the numerical radius is always an equivalent norm. Actually, the set of values of the numerical index was established by J. Duncan, C. McGregor, J. Pryce, and A. White [6], who proved that

$$
\begin{aligned}
\{n(X): X \text { complex Banach space }\} & =\left[\mathrm{e}^{-1}, 1\right] \\
\{n(X): X \text { real Banach space }\} & =[0,1] .
\end{aligned}
$$

Even before the name of numerical index was introduced, it was known that a Hilbert space of dimension greater than one has numerical index $1 / 2$ in the complex case, and 0 in the real case (see [14, §17]). $L$ - and $M$-spaces have numerical index 1 [6], a property shared by the disk algebra 5. Theorem 3.3], and by every Banach space nicely embedded into any $C_{b}(\Omega)$-space [37, Corollary 2.2] (even by every space that is semi-nicely embedded into any $C_{b}(\Omega)$-space [23, Corollary 2]). Very recently, approximations to the computation of the numerical index of the $L_{p}(\mu)$-spaces have been made [8, 9], and the exact computation of the numerical indices of the two-dimensional spaces whose unit balls are regular polygons appears in [25].

Let us mention here a couple of facts concerning the numerical index which will be relevant to our discussion. Let us fix a bounded linear operator $T$ on a Banach space $X$. It is a well-known result of the theory of numerical ranges (see [3, §9]) that

$$
\sup \operatorname{Re} V(T)=\lim _{\alpha \downarrow 0} \frac{\|\operatorname{Id}+\alpha T\|-1}{\alpha}
$$

and so,

$$
v(T)=\max _{\omega \in \mathbb{T}} \lim _{\alpha \downarrow 0} \frac{\|\mathrm{Id}+\alpha \omega T\|-1}{\alpha},
$$

where $\mathbb{T}$ stands for the unit sphere of the base field $\mathbb{K}(=\mathbb{R}$ or $\mathbb{C})$. On the one hand, we can deduce from the above formula that

$$
v(T)=\|T\| \quad \Longleftrightarrow \quad \max _{\omega \in \mathbb{T}}\|\operatorname{Id}+\omega T\|=1+\|T\|
$$


(see [27, Lemma 2.3]). On the other hand, it also implies that $v(T)=v\left(T^{*}\right)$, where $T^{*}$ is the adjoint operator of $T$, and it clearly follows that

$$
n\left(X^{*}\right) \leqslant n(X)
$$

for every Banach space $X$ (see [4 §32]). The question if this is actually an equality, which is certainly true for reflexive spaces, has been around from the beginning of the subject.

The main aim of this paper is to give a negative answer to the above question, i.e., we will show that the numerical index of the dual of a Banach space can be strictly smaller than the numerical index of the space.

The outline of the paper is as follows. In Section 2 we introduce a massiveness property for a Banach space called "lushness" which implies numerical index 1, and we prove that C-rich subspaces of $C(K)$-spaces satisfy it. Next, we use the above results in Section 3 to present examples of Banach spaces whose numerical index is strictly bigger than the numerical index of their duals, and other related counterexamples. Finally, we devote Section 4 to show a positive result: the dual of a Banach space having the Radon-Nikodým property (RNP for short) and numerical index 1 also has numerical index 1.

We finish this introduction by recalling some definitions and fixing notation.

Let $X$ be Banach space. Recall that $x_{0} \in B_{X}$ is said to be a denting point of $B_{X}$ if it belongs to slices of $B_{X}$ with arbitrarily small diameter. More precisely, for each $\varepsilon>0$ one can find a functional $x^{*} \in S_{X^{*}}$ and a positive number $\alpha$ such that the slice

$$
S\left(B_{X}, x^{*}, \alpha\right):=\left\{x \in B_{X}: \operatorname{Re} x^{*}(x)>1-\alpha\right\}
$$

contains $x_{0}$ and is contained in turn in the closed ball centered at $x_{0}$ with radius $\varepsilon$. If $X$ is a dual space and the functionals $x^{*}$ can be taken to be $w^{*}$-continuous, then we say that $x_{0}$ is a $w^{*}$-denting point. If $B$ is a subset of $X$, we write $\operatorname{co}(B)$ and $\overline{c o}(B)$ to denote, respectively, the convex and closed convex hull of $B$. Then, $\operatorname{co}(\mathbb{T} B)$ will be the absolutely convex hull of $B$. Finally, we denote by $\operatorname{ext}(A)$ the set of extreme points of the convex subset $A \subseteq X$.

\section{Lush spaces and C-Rich subspaces of $C(K)$}

A Banach space $X$ is an almost- $C L$-space if $B_{X}$ is the closed absolutely convex hull of every maximal convex subset of $S_{X}$. This notion was introduced by $\AA$. Lima 19, generalizing the concept of $C L$-space (the same definition without closure) given by R. Fullerton [11] in 1960. We refer to 29, 34 and references therein for recent results. Real and complex almost-CL-spaces have numerical index 1 (see [22, §4]). Actually, the basic examples of Banach spaces with numerical index 1 are known to be almost-CL-spaces (see [29] and [4. Theorem 32.9]). The next definition is a weakening of the concept of almost-CL-space which still implies numerical index 1. We will show later (Example 3.4) that this weakening is strict, giving in particular an example of a Banach space with numerical index 1 which is not an almost-CL-space.

Definition 2.1. We say that a Banach space $X$ is lush if for every $x, y \in S_{X}$ and every $\varepsilon>0$, there exists $y^{*} \in S_{Y^{*}}$ such that $y \in S\left(B_{X}, y^{*}, \varepsilon\right)$ and

$$
\operatorname{dist}\left(x, \operatorname{co}\left(\mathbb{T} S\left(B_{X}, y^{*}, \varepsilon\right)\right)\right)<\varepsilon .
$$

The (immediate) proof of the fact that almost-CL-spaces have numerical index 1 can be straightforwardly extended to lush spaces.

Proposition 2.2. Let $X$ be a lush Banach space. Then $n(X)=1$.

Proof. For $T \in L(X)$ with $\|T\|=1$, and $0<\varepsilon<1 / 2$ fixed, we take $x_{0} \in S_{X}$ such that $\left\|T x_{0}\right\|>1-\varepsilon$, and we apply the definition of lushness to $x_{0}$ and $y_{0}=\frac{T x_{0}}{\left\|T x_{0}\right\|}$ to get 
$y^{*} \in S_{Y^{*}}$ with $y_{0} \in S\left(B_{X}, y^{*}, \varepsilon\right)$ and $x_{1}, \ldots, x_{n} \in S\left(B_{X}, y^{*}, \varepsilon\right), \theta_{1}, \ldots, \theta_{n} \in \mathbb{T}$ such that a convex combination $v=\sum \lambda_{k} \theta_{k} x_{k}$ of elements $\theta_{1} x_{1}, \ldots, \theta_{n} x_{n}$ approximates $x_{0}$ up to $\varepsilon$. Then

$$
\left|y^{*}(T v)\right|=\left|y^{*}\left(y_{0}\right)-y^{*}\left(T\left(\frac{x_{0}}{\left\|T x_{0}\right\|}-v\right)\right)\right|>1-4 \varepsilon,
$$

but on the other hand $y^{*}(T v)$ is a convex combination of $y^{*}\left(\theta_{1} T x_{1}\right), \ldots, y^{*}\left(\theta_{n} T x_{n}\right)$. So there is an index $j$ such that

$$
\left|y^{*}\left(T x_{j}\right)\right|=\left|y^{*}\left(\theta_{j} T x_{j}\right)\right|>1-4 \varepsilon \text {. }
$$

Now, we have

$$
\begin{aligned}
\max _{\omega \in \mathbb{T}}\|\operatorname{Id}+\omega T\| & \geqslant \max _{\omega \in \mathbb{T}}\left|y^{*}\left([\operatorname{Id}+\omega T]\left(x_{j}\right)\right)\right| \geqslant \max _{\omega \in \mathbb{T}}\left|y^{*}\left(x_{j}\right)+\omega y^{*}\left(T x_{j}\right)\right| \\
& =\left|y^{*}\left(x_{j}\right)\right|+\left|y^{*}\left(T x_{j}\right)\right|>2-5 \varepsilon .
\end{aligned}
$$

Letting $\varepsilon \downarrow 0$ we deduce that $\max _{\omega \in \mathbb{T}}\|\operatorname{Id}+\omega T\|=1+\|T\|$ and therefore, $v(T)=\|T\|$.

Real or complex $C(K)$-spaces are almost-CL-spaces (actually, they are CL-spaces, see [29]) and therefore, lush. We now present a wide class of subspaces of $C(K)$ which are lush, but, as we will show in Example 3.4 they are not almost-CL-spaces in general.

Definition 2.3. Let $K$ be a compact Hausdorff space. A closed subspace $X$ of $C(K)$ is said to be $C$-rich if for every nonempty open subset $U$ of $K$ and every $\varepsilon>0$, there is a positive function $h$ of norm 1 with support inside $U$ such that the distance from $h$ to $X$ is less than $\varepsilon$.

Theorem 2.4. Let $K$ be a compact Hausdorff space and let $X$ be a $C$-rich subspace of $C(K)$. Then $X$ is lush and, therefore, $n(X)=1$.

Proof. We fix $x, y \in S_{X}$ and $\varepsilon>0$. We take $t_{0} \in K$ such that $\left|y\left(t_{0}\right)\right|=1$ and we write $a=x\left(t_{0}\right), b=y\left(t_{0}\right)$. Find an open subset $U$ of $K$ with $t_{0} \in U$ and such that

$$
|x(t)-a|<\varepsilon / 4 \quad \text { and } \quad|y(t)-b|<\varepsilon / 4
$$

for every $t \in U$. Finally, the C-richness of $X$ gives us a norm-one function $h: K \longrightarrow[0,1]$ with support inside $U$ and distance to $X$ less than $\varepsilon / 4$. Let $\tilde{h} \in S_{X}$ be a function with

$$
\|\tilde{h}-h\|<\varepsilon / 4 \text {. }
$$

Since $\|h\|=1$, there is $t_{1} \in U$ such that $h\left(t_{1}\right)=1$ and, by Eq. (1), we have

$$
\operatorname{Re} \bar{b} y\left(t_{1}\right) \geqslant \operatorname{Re} \bar{b} b-\left|y\left(t_{1}\right)-b\right|>1-\varepsilon / 4 .
$$

We claim that for every $\gamma \in S_{\mathbb{K}}(-a / b, 1)$, we have

$$
|a+\gamma b|=\left|\gamma-\left(-\frac{a}{b}\right)\right|=1
$$

and

$$
\|x+\gamma b h\| \leqslant 1+\varepsilon / 4 .
$$

Indeed, the first condition is clear. Let us prove the second one. If $t \notin U$, then

$$
|x(t)+\gamma b h(t)|=|x(t)| \leqslant 1 .
$$

If $t \in U$, then

$$
\begin{aligned}
|x(t)+\gamma b h(t)| & \leqslant|x(t)-a|+|a+\gamma b h(t)| \\
& \leqslant \varepsilon / 4+|a+\gamma b h(t)| .
\end{aligned}
$$

Since $h(t) \in[0,1]$, the number $a+\gamma y\left(t_{0}\right) h(t)$ is a convex combination of $a$ and $a+\gamma y\left(t_{0}\right)$, so $\left|a+\gamma y\left(t_{0}\right) h(t)\right| \leqslant 1$ by Eq. (4). 
Now, since $0 \in \operatorname{co}\left(S_{\mathbb{K}}(-a / b, 1)\right)$, we may find $\gamma_{1}, \gamma_{2} \in S_{\mathbb{K}}(-a / b, 1)$ and $\lambda \in[0,1]$ such that $0=\lambda \gamma_{1}+(1-\lambda) \gamma_{2}$. We consider

$$
y^{*}=\frac{\left.\bar{b} \delta_{t_{1}}\right|_{X}}{\left\|\left.\delta_{t_{1}}\right|_{X}\right\|} \in S_{X^{*}} \quad \text { and } \quad x_{i}=\frac{x+\gamma_{i} b \tilde{h}}{1+\varepsilon} \in X \quad(i=1,2),
$$

and we observe that Equations (2) and (5) give that $x_{1}, x_{2} \in B_{X}$.

Finally, $y \in S\left(B_{X}, y^{*}, 2 \varepsilon\right)$ by Eq. (3),

$$
\left\|x-\left(\lambda x_{1}+(1-\lambda) x_{2}\right)\right\|=\frac{\varepsilon}{1+\varepsilon}<\varepsilon,
$$

and, for $i=1,2$,

$$
\begin{aligned}
(1+\varepsilon)\left|y^{*}\left(x_{i}\right)\right| & \geqslant\left|x\left(t_{1}\right)+\gamma_{i} b \tilde{h}\left(t_{1}\right)\right| \geqslant\left|x\left(t_{1}\right)+\gamma_{i} b\right|-2\|h-\tilde{h}\| \\
& \geqslant\left|a+\gamma_{i} b\right|-\left|x\left(t_{1}\right)-a\right|-2\|h-\tilde{h}\|>1-\varepsilon / 4-\varepsilon / 2>1-\varepsilon,
\end{aligned}
$$

where we have used Equations (11), (2), and (4). Therefore, $x_{1}, x_{2} \in S\left(B_{X}, y^{*}, 2 \varepsilon\right)$.

When $K$ is perfect, our definition of C-richness coincides with the definition of richness given in [17 and thus every finite-codimensional subspace of $C(K)$ is C-rich (see [17, Proposition 1.2]). This is not always the case when $K$ has isolated points. Actually, the following result characterizes $\mathrm{C}$-rich finite-codimensional subspaces of $C(K)$. We recall that the support of an element $f \in C(K)^{*}$ (represented by the regular measure $\mu_{f}$ ) is

$$
\operatorname{supp}(f)=\bigcap\left\{C \subset K: C \text { closed, }\left|\mu_{f}\right|(K \backslash C)=0\right\} .
$$

Proposition 2.5. Let $K$ be a compact Hausdorff space and let $f_{1}, \ldots, f_{n} \in C(K)^{*}$. The subspace

$$
Y=\bigcap_{i=1}^{n} \operatorname{ker} f_{i}
$$

is $C$-rich if and only if $\bigcup_{i=1}^{n} \operatorname{supp}\left(f_{i}\right)$ does not intersect the set of isolated points of $K$.

Proof. We fix a nonempty open subset $U$ of $K$ and $\varepsilon>0$. If $\bigcup_{i=1}^{n} \operatorname{supp}\left(f_{i}\right)$ does not contain any isolated point of $K$, we may consider two cases. Case 1: $U$ contains an isolated point of $K$ (say, $\tau$ ). Then $h=\chi_{\{\tau\}} \in S_{C(K)}$ is a positive $U$-supported function which lies in $Y$, so $\operatorname{dist}(h, Y)=0<\varepsilon$. Case 2: $U$ does not contain isolated points of $K$. In this case one can find a sequence of disjoint open subsets $U_{n} \subset U$ and a sequence of positive $h_{n} \in S_{C(K)}$ with $\operatorname{supp}\left(h_{n}\right) \subset U_{n}$. Denote by $q: X \rightarrow X / Y$ the natural quotient map. Since $\left(h_{n}\right)$ tends weakly to 0 as $n \rightarrow \infty,\left(q\left(h_{n}\right)\right)$ tends weakly to 0 . But $X / Y$ is finite-dimensional, so $\left\|q\left(h_{n}\right)\right\|=\operatorname{dist}\left(h_{n}, Y\right) \rightarrow 0$ as well, and we can select $n \in \mathbb{N}$ with $\operatorname{dist}\left(h_{n}, Y\right)<\varepsilon$ and $\operatorname{supp}\left(h_{n}\right) \subseteq U_{n} \subset U$. So in both cases $Y$ is C-rich.

Conversely, suppose, for the sake of simplicity, that $\operatorname{supp}\left(f_{1}\right)$ contains an isolated point $t_{0} \in K$. Then, $\mu_{f_{1}}\left(\left\{t_{0}\right\}\right) \neq 0$ (if not, $t_{0} \notin \operatorname{supp}\left(f_{1}\right)$ ), and for every positive norm-one function $h$ with support inside $U$, one has $\operatorname{dist}\left(h, \operatorname{ker} f_{1}\right) \geqslant\left|\mu_{f_{1}}\left(\left\{t_{0}\right\}\right)\right|>0$. Hence $\operatorname{ker} f_{1}$ is not a C-rich subspace, and neither is $Y$.

\section{The countereXamples}

Let us recall that $c$ denotes the Banach space of all convergent scalar sequences $x=$ $(x(1), x(2), \ldots)$ equipped with the sup-norm. Evidently, $c$ is isometric to $C(K)$ where $K=$ $\mathbb{N} \cup\{\infty\}$ is the one-point compactification of $\mathbb{N}$. We are now ready for the main result of the paper. 
Example 3.1. There exists a Banach space $X$ such that $n(X)=1$ and $n\left(X^{*}\right)<1$. Indeed, we consider

$$
X=\left\{(x, y, z) \in c \oplus_{\infty} c \oplus_{\infty} c: \lim x+\lim y+\lim z=0\right\},
$$

which is a C-rich subspace of $c \oplus_{\infty} c \oplus_{\infty} c$ by Proposition 2.5] and, therefore, Theorem 2.4 gives us that $n(X)=1$. Let us prove that $n\left(X^{*}\right)<1$. We consider the closed subspace of $X$ given by

$$
Y=\left\{(x, y, z) \in c \oplus_{\infty} c \oplus_{\infty} c: \lim x=\lim y=\lim z=0\right\} .
$$

Since $Y$ is an $M$-ideal in $c \oplus_{\infty} c \oplus_{\infty} c$ (see [15. Example I.1.4(a)]), it is a fortiori an $M$ ideal in $X$ by [15. Proposition I.1.17], meaning that $Y^{\perp} \equiv(X / Y)^{*}$ is an $L$-summand of $X^{*}$. Therefore, $n\left(X^{*}\right) \leqslant n\left(Y^{\perp}\right)$ by [28, Proposition 1]. But $X / Y$ identifies with the twodimensional space

$$
\left\{(a, b, c) \in \ell_{\infty}^{(3)}: a+b+c=0\right\}
$$

which does not have numerical index 1 (in the real case, Remark 3.6 of [32] gives directly the result, since the unit ball of this space is a hexagon; the complex case follows routinely from Theorem 3.1 of the same paper).

Remark 3.2. In [31, Lemma 4.8] the reader may find a result which could be considered as contradictory with the above example. Let us recall that there is a concept of numerical range for elements of unital Banach algebras (see [3, Chapter 1], for instance). Given a Banach algebra $A$ with unit $u$, we define the algebra numerical range of an element $a \in A$ by

$$
V(A, a)=\left\{\varphi(a): \varphi \in A^{*},\|\varphi\|=\varphi(u)=1\right\} .
$$

We have then a corresponding algebra numerical radius $v(A, a)$ and the corresponding algebra numerical index $n_{\mathrm{a}}(A)$ of $A$. Given a Banach space $X$, if we consider the unital Banach algebra $A=L(X)$, it is well-known that

$$
V(L(X), T)=\overline{\mathrm{co}} V(T)
$$

for every $T \in L(X)$ [3, Theorem 9.4] and thus, $n(X)=n_{\mathrm{a}}(L(X))$. It follows from [31, Lemma 4.8] that

$$
n_{\mathrm{a}}(L(X))=n_{\mathrm{a}}\left(L(X)^{* *}\right)
$$

but, in general, $L\left(X^{* *}\right)$ does not coincide with $L(X)^{* *}$.

With just a little bit of work, Example 3.1 can be pushed to produce even better counterexamples.

\section{Examples 3.3.}

(a) There exists a real Banach space $X$ such that $n(X)=1$ and $n\left(X^{*}\right)=0$. Indeed, for every integer $n \geqslant 2$, we denote by $Z_{n}$ the 2-dimensional real normed space whose unit ball is the convex hull of the $(2 n)^{r m t h}$ roots of unity (i.e., its unit ball is a regular $2 n$-polygon such that one of its vertices is $(1,0))$. We observe that $Z_{n}$ is (isometric to) a subspace of $\ell_{\infty}^{(n)}$, and it is straightforward, following the lines of Example [3.1, to construct a C-rich subspace $X_{n}$ of $c \oplus_{\infty} c \oplus_{\infty} \cdots \oplus_{\infty} c$, and an $M$-ideal $Y_{n}$ of $X_{n}$ such that $X_{n} / Y_{n}$ is isometric to $Z_{n}$. It follows that $n\left(X_{n}\right)=1$ and $n\left(X_{n}^{*}\right) \leqslant n\left(Z_{n}\right)$. Finally, we consider

$$
X:=\left[\bigoplus_{n \geqslant 2} X_{n}\right]_{c_{0}},
$$

and we observe that $n(X)=1$ and $n\left(X^{*}\right) \leqslant n\left(X_{n}\right)$ for every $n \geqslant 2$. But this implies $n\left(X^{*}\right)=0$ since $n\left(X_{n}\right) \longrightarrow 0$ by [25], Theorem 5]. 
(b) There exists a complex Banach space $X$ such that $n(X)=1$ and $n\left(X^{*}\right)=1 /$ e. Let $Z$ be a two-dimensional complex normed space with numerical index 1/e (see [4] Lemma 32.2]). Then, we may find a family $\left\{Z_{n}\right\}$ of two-dimensional subspaces of $\ell_{\infty}^{(n)}$ such that the distance form $Z_{n}$ to $Z$ goes to 0 . Now, we follow the lines of the above example to get a Banach space $X$ such that $n(X)=1$ and $n\left(X^{*}\right) \leqslant n\left(Z_{n}\right)$ for every $n \in \mathbb{N}$. But the numerical index is continuous with respect to the distance between Banach spaces [10, Proposition 2], and so $n\left(X^{*}\right) \leqslant 1 / \mathrm{e}$.

As we have already mentioned at the beginning of Section 2 the main examples of Banach spaces with numerical index 1 are known to be almost-CL-spaces. Actually, it is proved in 23 that every Banach space with numerical index 1 and the RNP is an almost-CL-space and it satisfies that

$$
\left|x^{* *}\left(x^{*}\right)\right|=1 \quad\left(x^{* *} \in \operatorname{ext}\left(B_{X^{* *}}\right), x^{*} \in \operatorname{ext}\left(B_{X^{*}}\right)\right) .
$$

The Example 3.1 shows that these implications are not true in general, even for Asplund spaces, as the following result details. Recall that a boundary of $B_{X^{*}}$ is a subset $C$ of $B_{X^{*}}$ such that

$$
\|x\|=\max \{\operatorname{Re} f(x): f \in C\}
$$

for every $x \in X$. The classical boundary of $B_{X^{*}}$ is the set $\operatorname{ext}\left(B_{X^{*}}\right)$ (consequence of the Hahn-Banach and Krein-Milman Theorems).

Example 3.4. Let us consider the Banach space given in Example 3.1, i.e.,

$$
X=\left\{(x, y, z) \in c \oplus_{\infty} c \oplus_{\infty} c: \lim x+\lim y+\lim z=0\right\} .
$$

Then $X$ is an Asplund space, it is lush (and so $n(X)=1$ ), but the following properties hold.

(a) For every boundary $C \subset S_{X^{*}}$ of $B_{X^{*}}$, there exists $x^{*} \in C$ and $x^{* *} \in \operatorname{ext}\left(B_{X^{* *}}\right)$ such that $\left|x^{* *}\left(x^{*}\right)\right|<1$. Suppose that, on the contrary, $C$ is a boundary of $B_{X}$ such that $\left|x^{* *}\left(x^{*}\right)\right|=1$ for every $x^{*} \in C$ and every $x^{* *} \in \operatorname{ext}\left(B_{X^{* *}}\right)$. Now that $X$ is a space which does not contain $\ell_{1}, B_{X^{*}}$ is the norm-closed convex hull of $C$ [13, Theorem III.1]. Therefore, given $T \in L\left(X^{*}\right)$ and $\varepsilon>0$, we may find $x^{*} \in C$ and $x^{* *} \in \operatorname{ext}\left(B_{X^{* *}}\right)$ such that

$$
\left|x^{* *}\left(T x^{*}\right)\right|=\left\|T x^{*}\right\|>\|T\|-\varepsilon .
$$

This result together with the fact that $\left|x^{* *}\left(x^{*}\right)\right|=1$ gives that $v(T)>\|T\|-\varepsilon$; thus $n\left(X^{*}\right)=1$, a contradiction.

(b) In particular, there are $x^{*} \in \operatorname{ext}\left(B_{X^{*}}\right)$ and $x^{* *} \in \operatorname{ext}\left(B_{X^{* *}}\right)$ such that $\left|x^{* *}\left(x^{*}\right)\right|<1$.

(c) $X$ is not an almost-CL-space. This follows from (a) and [29, Lemma 3].

Remark 3.5. Let us observe that every Asplund space with numerical index 1 (in particular the above example) satisfies the following property: there is a subset $A$ of $S_{X^{*}}$ such that $B_{X^{*}}=\overline{\mathrm{co}}^{w^{*}}(A)$ and

$$
\left|x^{* *}\left(x^{*}\right)\right|=1 \quad\left(x^{* *} \in \operatorname{ext}\left(B_{X^{* *}}\right), x^{*} \in A\right) .
$$

This is a consequence of [20, Lemma 1], where $A$ is the set of all $w^{*}$-denting points of $B_{X^{*}}$. The above property is clearly sufficient for an arbitrary Banach space to have numerical index 1 (see [24, §1], for instance), but we do not know if it is also necessary without the Asplundness assumption.

Once we know that the numerical index of a Banach space and the one of its dual do not coincide, another natural question could be if two isometric preduals of a given Banach space should have the same numerical index. The answer is again negative as the following result shows. 
Example 3.6. There is a Banach space $Z$ with two isometric preduals $X_{1}$ and $X_{2}$ such that $n\left(X_{1}\right)$ and $n\left(X_{2}\right)$ are not equal. Indeed, let

$$
X_{1}=\left\{(x, y, z) \in c \oplus_{\infty} c \oplus_{\infty} c: \lim x+\lim y+\lim z=0\right\}
$$

and

$$
X_{2}=\left\{(x, y, z) \in c \oplus_{\infty} c \oplus_{\infty} c: x(1)+y(1)+z(1)=0\right\} .
$$

By Example $3.1 n\left(X_{1}\right)=1$. Since the two dimensional space

$$
\left\{(a, b, c) \in \ell_{\infty}^{(3)}: a+b+c=0\right\}
$$

is isometric to an $M$-summand of $X_{2}$, it follows that $n\left(X_{2}\right)<1$ (see [28, Proposition 1] and 32. Theorem 3.1]). Finally, the fact that $X_{1}^{*}$ and $X_{2}^{*}$ are isometric is straightforward.

\section{A positive Result}

As a straightforward application of the inequality (画), i.e., $n\left(X^{*}\right) \leqslant n(X)$, it is clear that $n(X)=n\left(X^{*}\right)$ for every reflexive space $X$. This equality also holds when $X$ is a Banach space such that $n\left(X^{*}\right)=1$, in particular when $X$ is an $L$ - or an $M$-space. Besides these elementary results, it is also true that $n(X)=n\left(X^{*}\right)$ when $X$ is a $C^{*}$-algebra or a von Neumann predual (see [16] and [18, pp. 202]).

We finish the paper by showing another particular case where inequality (因) becomes an equality.

Proposition 4.1. Let $X$ be a Banach space with the RNP. If $n(X)=1$, then $n\left(X^{*}\right)=1$.

Proof. By [20, Lemma 1], we have that $\left|x^{*}(x)\right|=1$ for every extreme point $x^{*}$ of $B_{X^{*}}$ and every denting point $x \in B_{X}$. Therefore, 7, Proposition 2.1] (or [35, Proposition 3.5]) gives us that

$$
\left|x^{* * *}(x)\right|=1
$$

for every $x^{* * *} \in \operatorname{ext}\left(B_{X^{* * *}}\right)$ and every denting point $x \in B_{X}$. Now, we fix $T \in L\left(X^{*}\right)$ and $\varepsilon>0$. Since $X$ has the RNP, $B_{X^{* *}}$ is the weak*-closed convex hull of the set of denting points of $B_{X}$, and we may find a denting point $x$ such that

$$
\left\|T^{*} x\right\|>\|T\|-\varepsilon .
$$

Then, we may find $x^{* * *} \in \operatorname{ext}\left(B_{X^{* * *}}\right)$ such that

$$
\left|x^{* * *}\left(T^{*} x\right)\right|=\left\|T^{*} x\right\|>\|T\|-\varepsilon .
$$

This fact, together with Eq. (6), implies that $\left\|T^{*}\right\|-\varepsilon \leqslant v\left(T^{*}\right)$. By letting $\varepsilon \downarrow 0$, we have

$$
\|T\|=\left\|T^{*}\right\|=v\left(T^{*}\right)=v(T) .
$$

We do not know if $n(X)=n\left(X^{*}\right)$ for every Banach space with the Radon-Nikodým property.

\section{REFERENCES}

[1] F. L. Bauer, On the field of values subordinate to a norm, Numer. Math. 4 (1962), 103-111.

[2] H. F. Bohnenblust and S. Karlin, Geometrical properties of the unit sphere in Banach algebras, Ann. of Math., 62 (1955), 217-229.

[3] F. F. Bonsall and J. Duncan, Numerical Ranges of Operators on Normed Spaces and of Elements of Normed Algebras, London Math. Soc. Lecture Note Series 2, Cambridge, 1971.

[4] F. F. Bonsall and J. Duncan, Numerical Ranges II, London Math. Soc. Lecture Note Series 10, Cambridge, 1973.

[5] M. J. Crabb, J. Duncan, and C. M. McGregor, Mapping theorems and the numerical radius, Proc. London Math. Soc. 25 (1972), 486-502.

[6] J. Duncan, C. McGregor, J. Pryce, and A. White, The numerical index of a normed space, $J$. London Math. Soc. 2 (1970), 481-488. 
[7] S. Dutta and T. S. S. R. K. Rao, On weak*-extreme points in Banach spaces, J. Convex Anal. 10 (2003), 531-539.

[8] E. Ed-Dari, On the numerical index of Banach spaces, Linear Algebra Appl. 403 (2005), 86-96.

[9] E. Ed-Dari and M. A. Khamsi, The numerical index of the $L_{p}$ space, Proc. Amer. Math. Soc., to appear.

[10] C. Finet, M. Martín, and R. Payá, Numerical index and renorming, Proc. Amer. Math. Soc. 131 (2003), 871-877.

[11] R. E. Fullerton, Geometrical characterization of certain function spaces. In: Proc. Inter. Sympos. Linear spaces (Jerusalem 1960), pp. 227-236. Pergamon, Oxford 1961.

[12] B. W. Glickfeld, On an inequality of Banach algebra geometry and semi-inner-product space theory, Illinois J. Math., 14 (1970), 76-81.

[13] G. Godefroy, Boundaries of a convex set an interpolation sets, Math. Ann. 277 (1987), 173-184.

[14] P. Halmos, A Hilbert space problem book, Van Nostrand, New York, 1967.

[15] P. Harmand, D. Werner, and D. Werner, $M$-ideals in Banach spaces and Banach algebras, Lecture Notes in Math. 1547, Springer-Verlag, Berlin 1993.

[16] T. Huruya, The normed space numerical index of $C^{*}$-algebras, Proc. Amer. Math. Soc. 63 (1977), $289-290$.

[17] V. M. Kadets and M. M. Popov, The Daugavet property for narrow operators in rich subspaces of $C[0,1]$ and $L_{1}[0,1]$, St. Petersburg Math. J. 8 (1997), 571-584.

[18] A. Kaidi, A. Morales, and A. Rodríguez-Palacios, Geometrical properties of the product of a $C^{*}$-algebra, Rocky Mountain J. Math. 31 (2001), 197-213.

[19] А. Lima, Intersection properties of balls in spaces of compact operators, Ann. Inst. Fourier, Grenoble 28 (1978), 35-65.

[20] G. López, M. Martín, and R. Payá, Real Banach spaces with numerical index 1, Bull. London Math. Soc. 31 (1999), 207-212.

[21] G. Lumer, Semi-inner-product spaces, Trans. Amer. Math. Soc. 100 (1961), 29-43.

[22] M. Martín, A survey on the numerical index of a Banach space, Extracta Math 15 (2000), 265-276.

[23] M. Martín, Banach spaces having the Radon-Nikodým property and numerical index 1, Proc. Amer. Math. Soc. 131 (2003), 3407-3410.

[24] M. Martín, The alternative Daugavet property for $C^{*}$-algebras and $J B^{*}$-triples, preprint. Available at http://arXiv.org/abs/math.FA/0411555

[25] M. Martín and J. Merí, Numerical index of some polyhedral norms on the plane, preprint.

[26] M. Martín, J. Merí, and A. Rodríguez-Palacios, Finite-dimensional Banach spaces with numerical index zero, Indiana U. Math. J. 53 (2004), 1279-1289.

[27] M. Martín and T. Oikhberg, An alternative Daugavet property, J. Math. Anal. Appl. 294 (2004), $158-180$.

[28] M. Martín and R. Payá, Numerical index of vector-valued function spaces, Studia Math. 142 (2000), 269-280.

[29] M. Martín and R. PayÁ, On CL-spaces and almost-CL-spaces, Ark. Mat. 42 (2004), 107-118.

[30] M. Martín and A. R. Villena, Numerical index and Daugavet property for $L_{\infty}(\mu, X)$, Proc. Edinburgh Math. Soc. 46 (2003), 415-420.

[31] J. Martínez-Moreno, J. F. Mena-Jurado, R. Payá-Albert, and A. Rodríguez-Palacios, An approach to numerical ranges without Banach algebra theory, Illinois J. Math. 29 (1985), 609-626.

[32] C. M. McGregor, Finite dimensional normed linear spaces with numerical index 1, J. London Math. Soc. 3 (1971), 717-721.

[33] T. Oikhberg, Spaces of operators, the $\psi$-Daugavet property, and numerical indices, Positivity (to appear).

[34] S. ReIsner, Certain Banach spaces associated with graphs and CL-spaces with 1-unconditonal bases, J. London Math. Soc. 43 (1991), 137-148.

[35] M. Sharir, Extremal structure in operator spaces, Trans. Amer. Math. Soc. 186 (1973), 91-111.

[36] O. Toeplitz, Das algebraische Analogon zu einem Satze von Fejer, Math. Z. 2 (1918), 187-197.

[37] D. Werner, The Daugavet equation for operators on function spaces, J. Funct. Anal. 143 (1997), $117-128$. 